\title{
Commentary: data sharing in South East Asia
}

\author{
Louis Grue and colleagues describe their experiences of sharing data in an international research \\ collaboration
}

\author{
Louis Grue clinical project manager ${ }^{1}$, Sophia Siddiqui deputy branch chief ${ }^{2}$, Direk Limmathurotsakul \\ assistant professor ${ }^{3}$, Armaji Kamaludi country coordinator for SEAICRN-Indonesia ${ }^{4}$, Muhammad \\ Karyana chair, INA-RESPOND Indonesia ${ }^{4}$, Chuen-Yen Lau medical officer ${ }^{2}$
}

\begin{abstract}
${ }^{1}$ Clinical Research Directorate/Clinical Monitoring Research Program, Leidos Biomedical Research, NCI Campus at Frederick, Frederick, Maryland 21702, USA; ${ }^{2}$ Collaborative Clinical Research Branch, Division of Clinical Research, National Institutes of Health/National Institute of Allergy and Infectious Disease, USA; ${ }^{3}$ Faculty of Tropical Medicine, Mahidol University/Mahidol Oxford Research Unit, Thailand; ${ }^{4}$ National Institute of Health Research and Development, Ministry of Health, Indonesia
\end{abstract}

The purpose of data sharing is to facilitate scientific discovery and improve public health, by providing research data to the scientific community quickly and easily. ${ }^{1}$ This improves efficiency of resource utilisation and enables data to be combined to achieve increased power. Sharing of data may also facilitate increased public trust in research and evidence based decisions. $^{2}$

There are, however, concerns that research participants will be identified or the data misused. ${ }^{34}$ In some low and middle income countries, where the idea of data sharing is not widely known or accepted, these concerns may have greater weight. ${ }^{56}$

The South East Asia Infectious Disease Clinical Research Network (SEAICRN) was a collaborative partnership which began in 2005 between hospitals and research institutions in South East Asia (box). Our experience with the network highlights some of the problems that can arise in collaborations, particularly in international environments.

\section{Including data sharing in protocols}

Researchers from different countries may have varying experience and requirements for data sharing. When we were planning to make our data open access, it became apparent that Indonesia had several regulations on data sharing that needed to be considered. Investigators from Thailand and Vietnam had differing ideas and understanding of what, how, and when data should be shared. Some investigators supported sharing the entire dataset in a repository while others preferred to limit it to the data that would be included in the planned manuscripts. Opinions also differed regarding unrestricted access to the data versus monitored and refereed access.

Although datasets can be held by the principal investigator and disseminated to those who want access, this increases the demands on the investigator long after the study has ended. The websites currently available to house datasets vary in cost (from free to around $\$ 3000$ ( $£ 2400$; $€ 2700$ ) and capabilities. The more expensive options allow the originators of the data to have more control over access. This has clear resource implications.

Investigators must not only determine how data are accessed but also the duration for which the data will remain available on these sites, and allocate funds. It is also imperative to identify someone from the outset as the custodian of the dataset, especially in a multicentre trial. This person is responsible for posting the data in a useable format, monitoring, answering queries, and maintaining the dataset, all of which require time and resources. In resource limited settings or when the study team will not be maintained these decisions can be particularly difficult, especially if not all sites want to participate in open access data sharing. The protocol should also include a contingency plan for open access data sharing in the event that study findings are not published within a certain period.

Addressing investigators' concerns can be difficult because experience is currently limited and a historical record of integrity is not yet established. Multiple levels of approval, including from high level government entities, may be needed to allow open access data sharing.

\section{Informed consent}

Even when the published data are deidentified, patients have a right to know how data from their study participation will be used and attitudes towards sharing of data vary. Some will see it as adding value to their study participation ${ }^{7}$ whereas others may view it as an invasion of privacy. They may want their information to be used only for a particular purpose. ${ }^{8}$

Information about plans to share data should be provided to potential participants in the consent. This should cover confidentiality as well as the plan for data storage and data sharing, ${ }^{9}$ including the potential risks. 


\section{Responsibilities of data users}

Standard processes for data sharing and acquisition would accelerate progress in developing platforms that facilitate better use of stored data. ${ }^{10}$ Currently the burden of open access data sharing is disproportionally placed on the investigators generating the data. It seems appropriate to establish ways to share these responsibilities with those using the dataset. This would help to reduce the anxiety and mistrust that less experienced investigators may have with the idea of open access data sharing and facilitate collaboration.

\section{Educating investigators about data sharing}

As with any new initiative, education is important in the understanding and acceptance of open access data sharing. Investigators with knowledge of the rationale, concerns, and requirements should have access to the tools to educate and inform their collaborators about the concept, its applications, and implementation.

Providing information in local languages where appropriate will help to build capacity and limit misunderstandings. Collaborators must be aware of resources that facilitate data sharing - for example, how to access data posted in electronic data warehouses and the costs. Awareness of journal requirements and all of the funder's policies will allow publication and successful competition for research support. This can also enable them to explain the need for sharing study data to their institutions, ethics committees, and other regulatory authorities. Success of open access data sharing as a strategy to promote research will ultimately be determined by whether it engenders more useful knowledge. We will need to evaluate whether open access data sharing has changed the landscape of research globally and what effect it has had on research, innovation, and, most importantly, trust.
We thank the South East Asia Infectious Disease Clinical Research Network governing board, steering committee, investigators, partners, and research support staff; the Indonesia Research Partnership on Infectious Diseases and its secretariat staff; the ministries of health of Thailand, Vietnam and Indonesia; and the study participants.

Competing interests: We have read and understood BMJ policy on declaration of interests and declare the project was funded by the National Cancer Institute, National Institutes of Health under Contract HHSN261200800001E. The content of this article does not necessarily reflect the views or policies of the Department of Health and Human Services.

Provenance and peer review: Not commissioned; not externally peer reviewed.

1 Modjarrad K, Moorthy VS, Millett P, Gsell PS, Roth C, Kieny MP. Developing global norms for sharing data and results during public health emergencies. PLoS Med 2016;13:e1001935. doi:10.1371/journal.pmed.1001935 pmid:26731342.

2 Cherico-Hsii S, Bankoski A, Singal P, et al. Sharing Overdose Data Across State Agencies to Inform Public Health Strategies: A Case Study. Public Health Rep 2016;131:258-63. doi:10.1177/003335491613100209 pmid:26957660.

3 Callaway E. Zika-microcephaly paper sparks data-sharing confusion. Nature 2016 Feb 12. doi:10.1038/nature.2016.19367

4 Drazen JM. Sharing individual patient data from clinical trials. N Engl J Med 2015;372:201-2. doi:10.1056/NEJMp1415160 pmid:25587944.

5 Hopkins C, Sydes M, Murray G, et al. UK publicly funded Clinical Trials Units supported a controlled access approach to share individual participant data but highlighted concerns. $J$ Clin Epidemiol 2016;70:17-25. doi:10.1016/j.jclinepi.2015.07.002 pmid:26169841.

6 Rice S. Doubters ask if IOM report will foster more sharing of clinical trial data. Mod Healthc 2015;45:10.pmid:25671895.

7 Darquy S, Moutel G, Lapointe AS, et al. Patient/family views on data sharing in rare diseases: study in the European LeukoTreat project. Eur J Hum Genet 2016;24:338-43. doi:10.1038/ejhg.2015.115 pmid:26081642.

8 Dheensa S, Fenwick A, Lucassen A. "Is this knowledge mine and nobody else's? I don't feel that." Patient views about consent, confidentiality and information-sharing in genetic medicine. J Med Ethics 2016;42:174-9. doi:10.1136/medethics-2015-102781 pmid: 26744307.

9 Australian National Data Service. Ethics, consent, and data sharing. http://www.ands.org. au/guides/ethics-consent-and-data-sharing

10 Geifman N, Bollyky J, Bhattacharya S, Butte AJ. Opening clinical trial data: are the voluntary data-sharing portals enough?BMC Med 2015;13:280. doi:10.1186/s12916-0150525-y pmid:26560699.

11 Taichman DB, Backus J, Baethge C, et al. Sharing clinical trial data. BMJ 2016;532:i255. doi:10.1136/bmj.i255 pmid:26790902.

Published by the BMJ Publishing Group Limited. For permission to use (where not already granted under a licence) please go to http://group.bmj.com/group/rights-licensing/ permissions 


\section{Case study: South East Asia Infectious Disease Clinical Research Network}

The South East Asia Infectious Disease Clinical Research Network (SEAICRN) was a collaborative partnership that began in 2005 between hospitals and research institutions in South East Asia. The network initiated a three year multicentre study in 2013, before open access data was widely required. The network became aware of open access data sharing guidelines ${ }^{11}$ in the last year of the study. The executive committee, comprised of representatives from each participating country, decided to amend the protocol to include data sharing. When local ethics committees processed the amendment, they raised concerns about how much patients needed to know. Given that this decision was made after all patients had been recruited, it was not feasible to go back and get amended consent.

Collaborators in Thailand and Indonesia had concerns about implementation of data sharing, and Indonesia has regulations for the sharing of data and samples. SEAICRN investigators were concerned about losing control of their data as well as inappropriate use of the data without their knowledge.

Although sharing of the entire study dataset was initially discussed, the team compromised on sharing only the data presented in the publication via an open access website. It agreed that data from research sites not amenable to open access would not be included in the publication.

Several options for sharing the data were considered. The group decided to use Figshare, which allows free posting of datasets and collects information on who accesses the data but does not allow for controlled access.

Several meetings were conducted to discuss and explain different aspects of data sharing and educational material was provided to

collaborators. A data sharing agreement was established within the network. With the increased understanding of open access data sharing and the motivation to be part of the primary study publications, Indonesia agreed to share data. Indonesia and one of the ethics committees in Thailand wanted to be made aware each time the data were accessed or used by investigators outside the network. Network collaborators agreed that a data sharing plan should be included in the protocol for future projects. 